\title{
Multi-Plane PIV Measurements in a Gasoline Direct Injection Engine
}

\author{
Li Shen ${ }^{1}$, Christopher Willman ${ }^{1}$, Richard Stone ${ }^{1}$, \\ Thomas Lockyer $^{2}$, Rachel Magnanon ${ }^{2}$ and Giuseppe Virelli ${ }^{2}$ \\ ${ }^{1}$ University of Oxford ${ }^{2}$ Jaguar Land Rover
}

\begin{abstract}
The flows in-cylinder have a profound effect on the mixture preparation and subsequent combustion in all engines. These flows are highly three-dimensional in nature and information from multiple planes is required to characterise the flow dynamics. The flow measurements reported here are from three orthogonal planes in an optical access engine that is based on the Jaguar Land Rover AJ200 Gasoline Direct Injection (GDI) engine. Particle Image Velocimetry (PIV) measurements have been taken every $5^{\circ} \mathrm{CA}$ from the start of induction to the end of compression. Data have been obtained from 300 cycles for separate experiments measuring flows in the tumble plane, the swirl plane and the cross-tumble plane. Vector comparison metrics are used to quantitatively compare ensemble averaged PIV flow fields to Computational Fluid Dynamics (CFD) simulations across each plane in terms of both the velocity magnitude and direction. These quantitative metrics enable identification of regions of interest and differences in flow characteristics across a range of engine operating conditions. Combination of the PIV vector fields from all three planes into composite flow fields enables the interaction between the flows in the three planes to be visualised. During induction the intake jet is visible in all three planes, while the swirl plane measurements show a pair of counter-rotating vortices either side of the intake jet and the tumble plane shows the development of the tumble flow. During compression the center of the tumble vortex traverses the cylinder leading to flow reversal in the swirl and cross tumble measurement planes.
\end{abstract}

\section{Introduction}

Development of internal combustion engines needs to continue so as to meet the ever increasingly demanding emissions legislation while at the same time seeking better fuel economy [1], [2]. Fundamental to this is understanding the complex in-cylinder flows that then determine the mixture preparation and the subsequent combustion processes. Computational Fluid Dynamics (CFD) is now widely used to simulate the flow processes and combustion in ICEs to improve the design of new combustion systems [3]-[5]. However, validation of the modelling is crucial, and a starting point has to be to ensure that the flow predictions are correct. Particle Image Velocimetry (PIV) is a widely used experimental technique for nonintrusive measurement of in-cylinder air motion in two or three dimensions [6]-[10]. This requires comprehensive optical access [11]-[15], but this can be achieved using a Bowditch piston design that incorporates a window [16].

The challenge is then to make comparisons between the predicted and measured vector fields without recourse to the inspection of individual flow fields. The quality of match between vector fields is frequently quantified using a metric such as the Relevance Index (RI) that compares the alignment of the vectors [17]-[19]. It is also necessary to compare the magnitude of the velocity predictions [20], [21].

However, the RI can be overly sensitive to the alignment of regions of low velocity such as the tumble vortex center, where even small differences in the locations of the vortex center between the modelled and measured data can result in very low values of the RI. Recent work ([22], [23]) has used a weighted function of the local velocity to provide a Weighted Relevance Index (WRI). The same type of approach has been applied to comparison of the magnitudes with a Weighted Magnitude Index (WMI). These metrics can also be normalized and combined into a single Combined Magnitude and Relevance Index (CMRI). This enables data sets with anomalies to be readily identified, and these cases can then be further investigated by applying the WMI and WRI metrics to establish whether the anomaly is due to misalignment or differences in magnitude.

\section{Experimental apparatus and procedure}

\section{Optical Engine}

PIV measurements were made in three orthogonal planes of a singlecylinder, optically accessible GDI engine with a pent-roof combustion chamber closely based on a recent production engine. This engine was operated under motored conditions and the spark plug was removed to minimize background illumination from scattered laser light. Engine specifications are given in Table 1 and Table 2.

\section{Table 1 Engine specification}

\begin{tabular}{ll}
\hline Bore $(\mathrm{mm})$ & 85 \\
Stroke $(\mathrm{mm})$ & 90 \\
Cylinder capacity $\left(\mathrm{cm}^{3}\right)$ & 512 \\
Compression ratio & 11 \\
Valves per cylinder & 2 intake, 2 exhaust \\
Intake air temperature $(\mathrm{K})$ & 318 \\
Coolant temperature $(\mathrm{K})$ & 298 \\
\hline
\end{tabular}

Table 2. Engine operating conditions

\begin{tabular}{|l|l|l|l|l|l|}
\hline $\begin{array}{l}\text { Test } \\
\text { condition }\end{array}$ & $\begin{array}{l}\text { Engine } \\
\text { speed } \\
(\mathrm{rpm})\end{array}$ & $\begin{array}{l}\text { Intake } \\
\text { pressure } \\
(\mathrm{kPa})\end{array}$ & $\begin{array}{l}\dot{V}_{\text {air }} \\
(\mathrm{L} / \mathrm{s})\end{array}$ & $\begin{array}{l}\text { IVO/IVC } \\
\left({ }^{\circ} \text { aTDC }\right)\end{array}$ & $\begin{array}{l}\text { EVO/EVC } \\
\left({ }^{\circ} \text { aTDC }\right)\end{array}$ \\
\hline $\mathrm{T} 1$ & 1500 & 80 & 1.57 & $327 /-259$ & $143 /-309$ \\
\hline $\mathrm{T} 2$ & 1500 & 95 & 3.65 & $330 /-243$ & $117 /-335$ \\
\hline
\end{tabular}

Optical access to the combustion chamber including the pent-roof is provided by a transparent annulus with a height varying between 25 and $39 \mathrm{~mm}$. Additionally, a quartz piston window $46 \mathrm{~mm}$ in diameter within the Bowditch piston provided access from below for either 
imaging or the laser sheet [Figure 1]. Excess background scatter was reduced by painting the rear internal surface of the transparent annulus, the valve edges and the cylinder head with matt black paint.

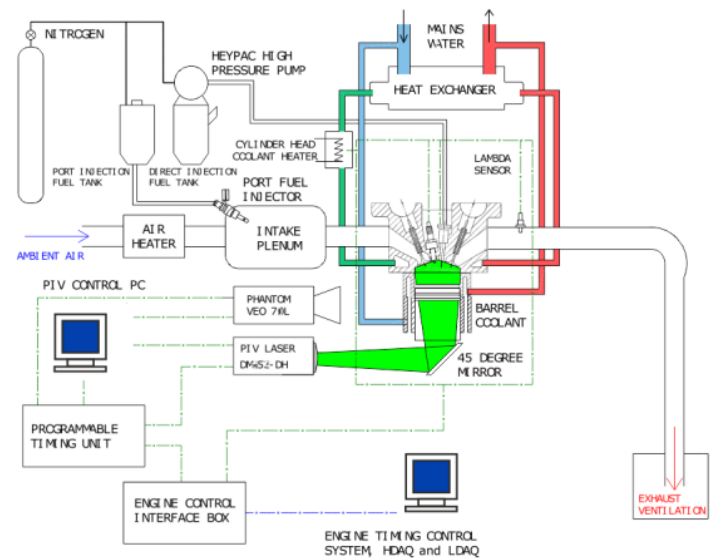

Figure 1 Experimental setup of the optical engine and PIV system (optics arrangement for tumble plane measurement are shown here as example).
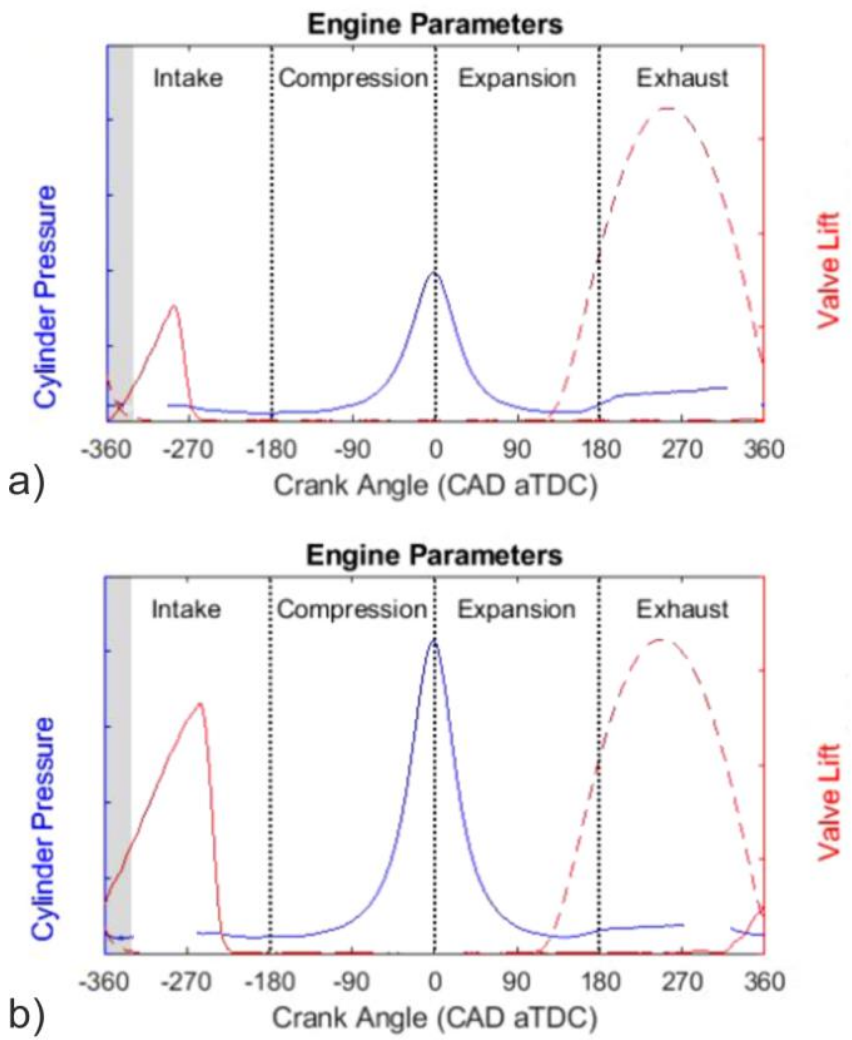

Figure 2 Intake and exhaust valve lift profiles and in-cylinder pressure traces for tests conditions a) $\mathrm{T} 1$ and b) T2.

\section{PIV System}

PIV measurements were made in

- The central tumble plane, a plane orthogonal to the crankshaft axis, but offset $1 \mathrm{~mm}$ away from the flywheel to minimize the strong scatter from the centrally located fuel injector tip.
- The central cross tumble plane which was orthogonal to the central tumble plane and had no offset from the cylinder axis.

- The swirl plane which was parallel to and $3.5 \mathrm{~mm}$ below the lower face of the cylinder head (firing deck).

PIV measurements were performed every $5^{\circ} \mathrm{CA}$ from $330^{\circ} \mathrm{bTDC}$ in the intake stroke to $30^{\circ} \mathrm{bTDC}$ at the end of the compression stroke. At each test condition, three experimental runs of 100 cycles were performed to build a 300-cycle set which was then averaged to produce the ensemble mean PIV data for comparison to CFD.

Droplets of olive oil approximately $0.2-0.4 \mu \mathrm{m}$ in diameter were introduced into the intake plenum by a LaVision aerosol generator controlled by a mass flow controller to achieve an iteratively optimized seeding density within the combustion chamber.

The oil droplets were illuminated by pulsed laser sheets, $1 \mathrm{~mm}$ thick and up to $60 \mathrm{~mm}$ wide at the measurement region, formed from the output of a diode-pumped, double cavity Nd:YLF laser (Photonics Industries DM20-527-DH) operating at $527 \mathrm{~nm}$ using a spherical lens telescope and a concave cylindrical lens with a focal length of -20 $\mathrm{mm}$. Each laser cavity provided a pulse energy of approximately 17 $\mathrm{mJ}$ at a repetition rate of $1.8 \mathrm{kHz}$. At every measurement timing within the engine cycle, the time separation between the pulses produced by each cavity was independently set using a LaVision Programmable Timing Unit (PTU v10) to minimize the loss of individual particle images from the interrogation window. This increases the dynamic range of the velocity measurements compared to a fixed delay system by optimizing the oil droplets' displacements between frames to suit the varying flow velocities throughout the cycle [24].

Images of the oil droplets were recorded using a Phantom VEO 710L high-speed 12-bit digital CMOS camera with a resolution of $1280 \mathrm{x}$ 800 pixels using a Nikon $50 \mathrm{~mm}$ f/1.4 lens working at f/4. During operation of the aerosol generator, build up of a layer of oil on the inner surface of the transparent annulus for some test conditions limited the number of cycles recorded per experimental run to 100 . The measurement rate of every $5^{\circ} \mathrm{CA}$ and range of 330 to $30^{\circ} \mathrm{bTDC}$ was selected due to the trade-off between the achievable frame rate and crank angle range for the 6000 image pairs capacity of the onboard camera memory.

The detrimental effects of astigmatism when imaging through the curved surface of the transparent annulus were minimized by adjusting the working distance of the camera to achieve equal defocus in the horizontal and vertical directions as suggested by Reuss et al. [25]. This slight defocus of the droplet images also helps to avoid peak-locking errors during calculation of the droplet displacements [26].

All image acquisition and processing was performed using LaVision $\mathrm{DaVis}$ 8.4.0 software. Distortion correction and image registration was achieved using an image of a grid of uniformly spaced dots.

The raw images were pre-processed using a sliding average filter across cycles to remove scattered light from static sources such as the intake valves and cylinder walls. A static digital mask was applied to ensure only oil droplet images were used to calculate velocities. Following a min-max filter, a multi-pass cross-correlation algorithm starting with a window size of $128 \times 128$ pixels and decreasing to $32 \times 32$ pixels for the final three passes was used to calculate the displacement of droplet images. For the tumble, cross-tumble and swirl planes respectively, this gave a spatial resolution of $1.91,1.69$ and $2.04 \mathrm{~mm}$ with a vector spacing of $0.96,0.84$ and $1.02 \mathrm{~mm}$ due to 
the $50 \%$ overlap between interrogation windows. Spurious vectors were removed from the calculated vector fields by deleting any vector with a correlation peak ratio (the ratio of the highest correlation peak to the second highest) of less than 1.8 and by application of the universal outlier detection method [27]. Only first choice vectors were included in the final vector fields, corresponding to approximately $99 \%$ of all vectors output from the PIV calculations. MATLAB was used for the analysis of the resulting vector fields.

The 2-dimensional 2-component (2D2C) PIV measurements in this work do not provide the out of plane component of velocity for the three planes of interest. As such, the CFD data has been restricted to the equivalent 2-components within each plane and all discussion of velocity directions and magnitudes refers to in-plane velocity only.

\section{Numerical Setup}

A commercially available CFD package, StarCD, was used for the RANS simulations of the GDI engine. The computational domain for the simulations is shown in Figure 3.

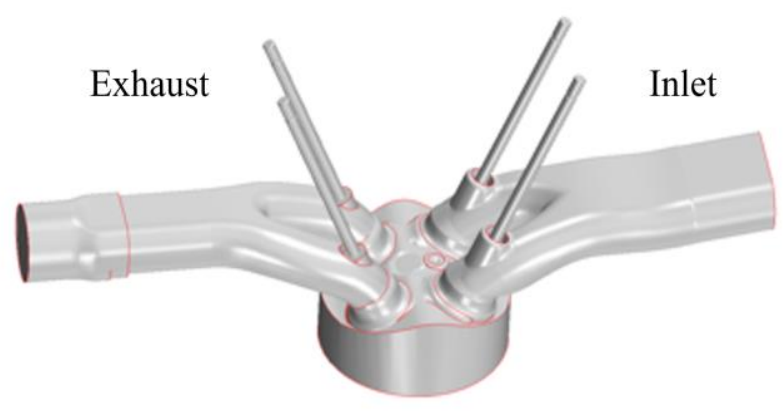

Figure 3. Image of the computational domain used for the RANS simulations

Boundary conditions for the simulations were provided by experimental data from the optical engine. Temperature and dynamic pressure measurements at the intake and exhaust ports were used as inputs along with the valve lift profiles [Figure 2], that use early inlet valve closure to reduce the part load pumping losses.

Dynamic meshing was used with an overall minimum cell size of 0.7 mm except for the valve curtain area for which the mesh was refined to $0.3 \mathrm{~mm}$. Each simulation contained approximately 3.3 million cells and utilized a maximum timestep of $0.1^{\circ} \mathrm{ca}$. Turbulence was modelled using the standard RNG k- $\varepsilon$ model and a modified Angelberger model was used to model heat transfer.

In order to match the simulated in-cylinder pressures to the experimental pressure traces [Figure 2], the crevice length (defined as the distance between the top land and the top piston ring) was varied for each condition. There was no further tuning of the physical models. The CFD package used linear interpolation between the cell locations to output velocity vectors at the locations of the PIV measurements, due to the minimum cell spacing of $0.7 \mathrm{~mm}$. Comparisons between the tumble ratio predicted by the CFD and the ensemble averaged PIV values have been presented already [23].

\section{Quantitative Metrics for Comparison of Vector Fields}

For details of the WRI, WMI and CMRI metrics and their application to analysis of flow fields the reader is directed to [22], [23]. A brief summary is provided here for convenience.

The Weighted Relevance Index incorporates a misalignment penalty based on the standard RI that is calculated at each spatial location for a pair of flow fields, $\left(1-\operatorname{RI}\left(x_{i}, z_{j}\right)\right) / 2$. This penalty is then weighted by the normalized local velocity magnitude of each field which suppresses the penalty for small local velocities. For a given angular difference this weighting assigns more importance to misaligned high velocity vectors (a large absolute velocity difference) than misaligned low velocity vectors. The WRI is defined as

$$
\begin{aligned}
\operatorname{WRI}\left(x_{i}, z_{j}\right)= & \left(\frac{1-\operatorname{RI}\left(x_{i}, z_{j}\right)}{2}\right) \\
& \times\left(\frac{Q_{A}\left(x_{i}, z_{j}\right)}{\operatorname{median}\left(Q_{A}\right)}\right) \\
& \times\left(\frac{Q_{B}\left(x_{i}, z_{j}\right)}{\operatorname{median}\left(Q_{B}\right)}\right)
\end{aligned}
$$

Where: $\quad Q_{A}(x, z)$ is the magnitude field of vector field $\boldsymbol{q}_{A}(x, z)$ such that $Q_{A}\left(x_{i}, z_{j}\right)=\left|\boldsymbol{q}_{A}\left(x_{i}, z_{j}\right)\right|$ with spatial coordinates $x_{i}$ and $z_{j}$. Here median $\left(Q_{A}\right)$ is defined as the median value of all elements (including duplicate values) of the magnitude field $Q_{A}$.

The normalization factor is chosen to be the median of the velocity magnitudes across the corresponding flow field for robustness against spurious vectors or small pockets of high velocities determining the weighting factor for the entire field.

The Weighted Magnitude Index is a method for quantifying differences in velocity magnitudes via normalization by the median velocity magnitude across both fields to produce a dimensionless metric, independent of the alignment of the vectors. Vectors with the same magnitude produce a WMI of zero, while vectors with large magnitude differences produce values of WMI greater than one.

The Weighted Magnitude Index (WMI) is defined as

$$
\operatorname{WMI}\left(x_{i}, z_{j}\right)=\frac{\left|Q_{A}\left(x_{i}, z_{j}\right)-Q_{B}\left(x_{i}, z_{j}\right)\right|}{\operatorname{median}\left(Q_{A}, Q_{B}\right)}
$$

Where: median $\left(Q_{A}, Q_{B}\right)$ is defined as the median of all elements (including duplicate values) of the magnitude fields $Q_{A}$ and $Q_{B}$.

Similarly to the WRI, the normalization factor has been chosen to be the median velocity magnitude across both vector fields A and B. This provides robustness of the normalization against spurious vectors or small pockets of high or low velocity as before.

The WRI and WMI often have very different distributions of values for a given set of flow field comparisons. In order to combine both the WRI and WMI into a single metric which accounts for differences in both alignment and magnitude, the CMRI, the ranges of the WRI and WMI are normalized to ensure the central $96 \%$ of values lie within the range 0 to 1 . 


$$
f_{\text {norm }}=\frac{f-f_{\text {low }}}{f_{\text {high }}-f_{\text {low }}}
$$

Where: $f_{\text {low }}$ is the threshold value of the WRI (or WMI) below which the best matched $2 \%$ of values occur. $f_{\text {high }}$ is the threshold value of the WRI (or WMI) above which the worst matched $2 \%$ of values occur.

This rescaling allows the Combined Magnitude and Relevance Index to be defined as

$$
\mathrm{CMRI}=\frac{\mathrm{WRI}_{\text {norm }}+\mathrm{WMI}_{\text {norm }}}{2}
$$

In this way the CMRI values retain sensitivity to both alignment and magnitude differences relevant to the flow fields of interest by ensuring neither the WRI and WMI metric values dominate the contribution to the CMRI. Throughout this paper, the normalized values $\mathrm{WRI}_{\text {norm }}$ and $\mathrm{WMI}_{\text {norm }}$ are presented when quantifying alignment or magnitude differences for ease of comparison to the CMRI values.

\section{Results}

\section{All planes - Isometric views}

Isometric views of the PIV and CFD flow fields across the tumble, cross-tumble and swirl planes are presented for six timings covering the intake (330 $\left.\mathrm{bTDC}, 300^{\circ} \mathrm{bTDC}, 200^{\circ} \mathrm{bTDC}\right)$ and compression $\left(140^{\circ} \mathrm{bTDC}, 80^{\circ} \mathrm{bTDC}, 40^{\circ} \mathrm{bTDC}\right)$ strokes in Figure 4 and Figure 5 for the test conditions $\mathrm{T} 1$ and $\mathrm{T} 2$ respectively.

On visual inspection there appears to be in most cases a good overall match between the experimental and simulated flow fields, with consistency in the structures of the in-cylinder flow across all three planes, for each crank angle and for both test conditions.

At $330^{\circ} \mathrm{bTDC}$ the PIV intake jet is visible in the tumble plane for both T1 [Figure 4] and T2 [Figure 5] and the directed intake runner, intended to generate high levels of tumble, gives a significant component of the flow in the x-direction from inlet to exhaust which results in the generation of a pair of counter-rotating vortices in the swirl plane.

At $300^{\circ}$ bTDC the intake jet is well established in all cases. The crosstumble and swirl planes show lower velocities except for a region of horizontal flow across the piston for the T1 PIV tumble plane flow field which is qualitatively not apparent for T2 PIV data or the CFD flow fields at either condition.

The flow fields at $200^{\circ} \mathrm{bTDC}, 140^{\circ} \mathrm{bTDC}$ and $80^{\circ} \mathrm{bTDC}$ show the evolution of the main tumble motion as the tumble vortex center moves from inlet to exhaust over the course of the late intake and early compression strokes. In-plane velocities for the cross-tumble plane are low for these timings due to the dominant flow motion being out of plane.

At $50^{\circ} \mathrm{bTDC}$ the rising piston causes the tumble vortex center to intersect the swirl measurement plane resulting in low in-plane velocities.
The RANS simulation has achieved a good qualitative match with the ensemble mean PIV measurements. However, some differences are apparent such as the increased velocity magnitudes within the tumble plane CFD flow fields, small differences in the tumble vortex center location between PIV and CFD and the extent of the intake jet for the T1 test condition. Discrepancies between the CFD and PIV jet flows can be a result of oscillations in the jet that are averaged-out when the PIV data is ensemble averaged, leading to lower peak velocities and a broader jet.

Quantitative comparisons provide greater insight into which flow features are well represented by CFD and which show differences to the experiments and have the potential to inform improvements to the simulation of these in-cylinder flows.

Before application of the WRI, WMI and CMRI metrics to quantify the differences between the PIV and CFD flow fields, the PIV flow fields across each of the three planes must be checked for consistency as PIV measurements for each plane were recorded with an interval of several months.

\section{PIV multi-plane validation}

At the intersection of the tumble and cross-tumble planes, the high downwards velocity present in the cross-tumble plane is not present in the tumble plane for the PIV flow field at $300^{\circ} \mathrm{bTDC}$ [Figure 4]. This suggests a change in the shape of the highly 3-dimensional intake jet between measurements for T1, perhaps a result of high sensitivity to the exact intake conditions of the nominally identical tumble and cross-tumble experiments.

The intersection of the three planes offers an opportunity to quantify the consistency between the three PIV experiments at each test condition as each pair of planes shares a single component of velocity along the line of intersection. The comparison of commoncomponent velocities along the intersections of the three planes are shown in [Figure 6] for $300^{\circ}$ bTDC.

The low load T1 test condition is poorly matched along the intersection of the cross-tumble plane with both other planes at $300^{\circ} \mathrm{bTDC}$. The trend of the velocity profile is consistent for the comparison of swirl and cross-tumble, albeit with an offset in both position and magnitude. The tumble and swirl plane common components are well matched, implying the discrepancy arises from the cross-tumble PIV measurement at T1.

This suggests that while the trends in differences identified between PIV and CFD for T1 are most likely valid, precise comparison of the location of the intake jet is affected by variation of the in-cylinder flow for the cross-tumble measurement. This effect will be most pronounced at crank angles for which the intake valves are open as the intake jet is highly fluctuating from cycle-to-cycle and may be more sensitive to the precise intake conditions. At later crank angles the in-cylinder flow is more stable, such as $140^{\circ}$ bTDC when the tumble motion is well-established, and T1 shows a good match of the common component of velocity between all three planes [Figure 7].

The higher load T2 test condition is well matched across all planes for PIV during both intake [Figure 6] and compression [Figure 7], implying that the variation of in-cylinder intake flow between planes for $\mathrm{T} 1$ is likely to be a consequence of the high variability of the incylinder flow during intake at such a low load condition, compared to the high load $\mathrm{T} 2$ which remained consistent over the same period of time. 

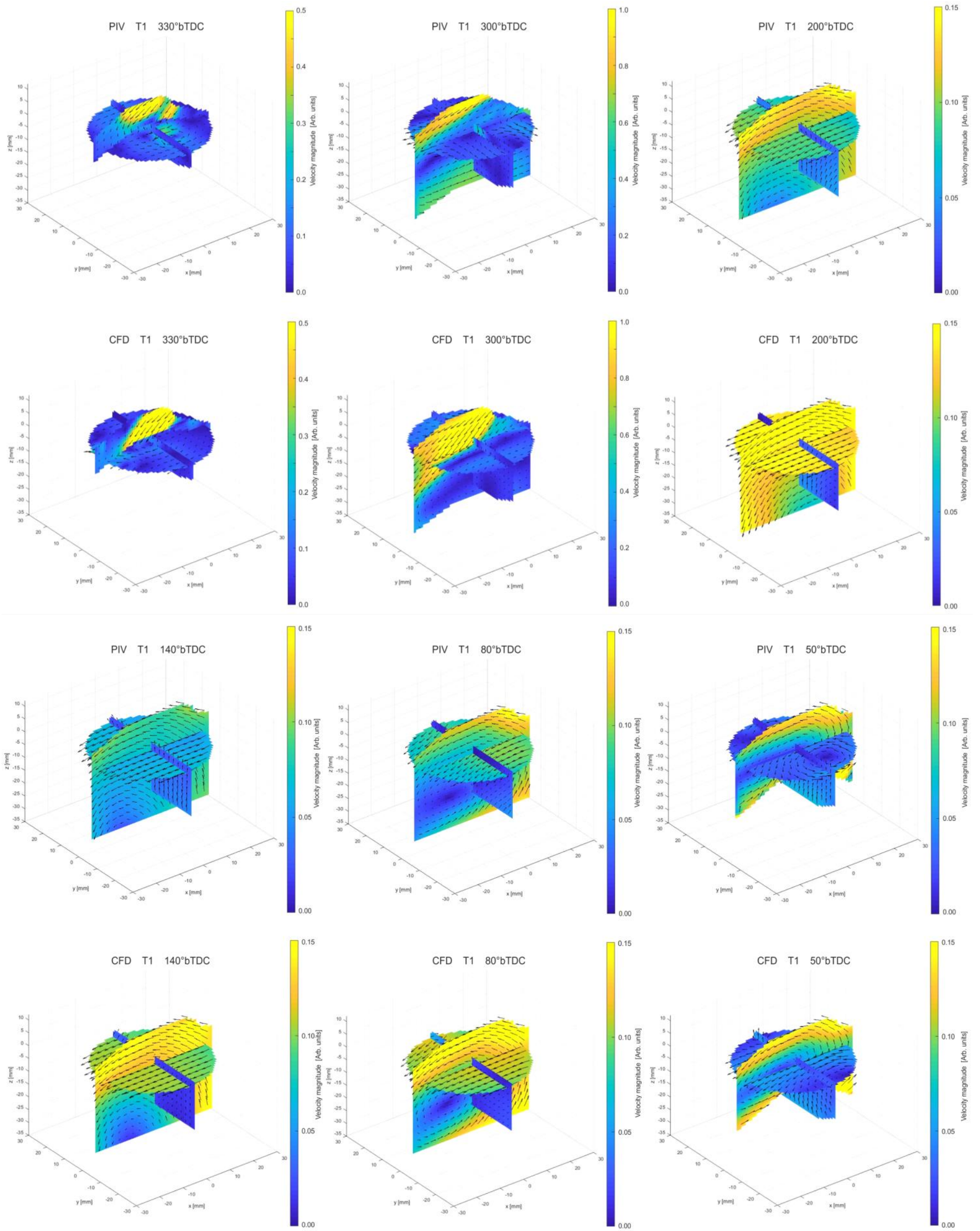

Figure 4 Isometric view of 2-component in-plane velocities for PIV and CFD flow fields in all three planes for T1.

Page 5 of 17 

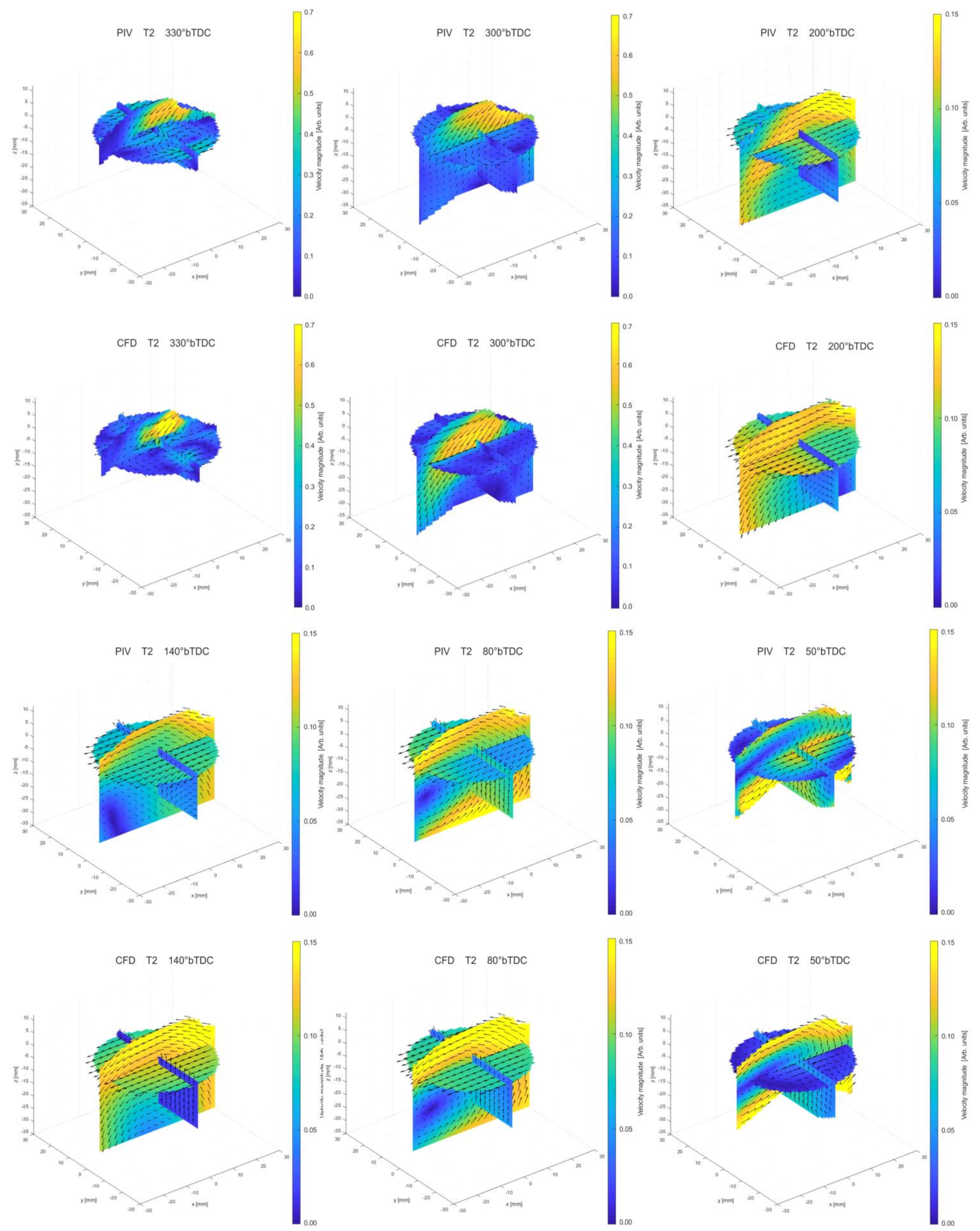

Figure 5 Isometric view of 2-component in-plane velocities for PIV and CFD flow fields in all three planes for T2.

Page 6 of 17 

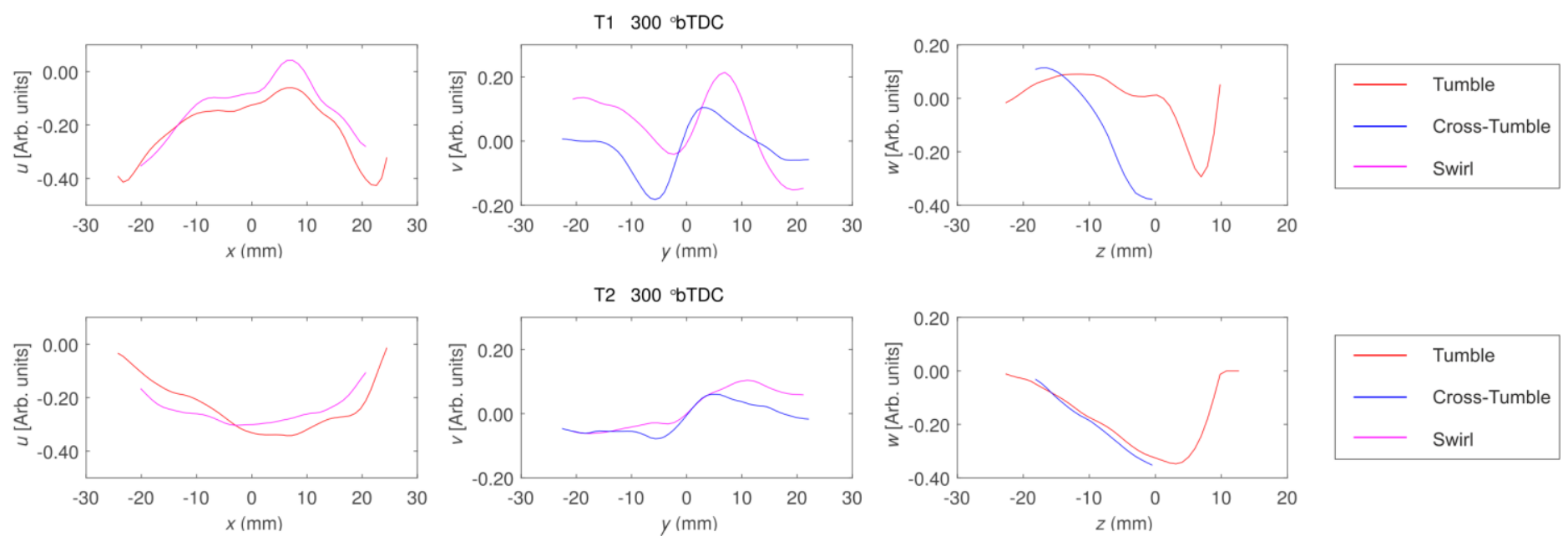

Figure 6 Comparison of common-component PIV velocities along the intersections of the three planes (Tumble, Cross-Tumble, Swirl) at $300^{\circ} \mathrm{bTDC}$.
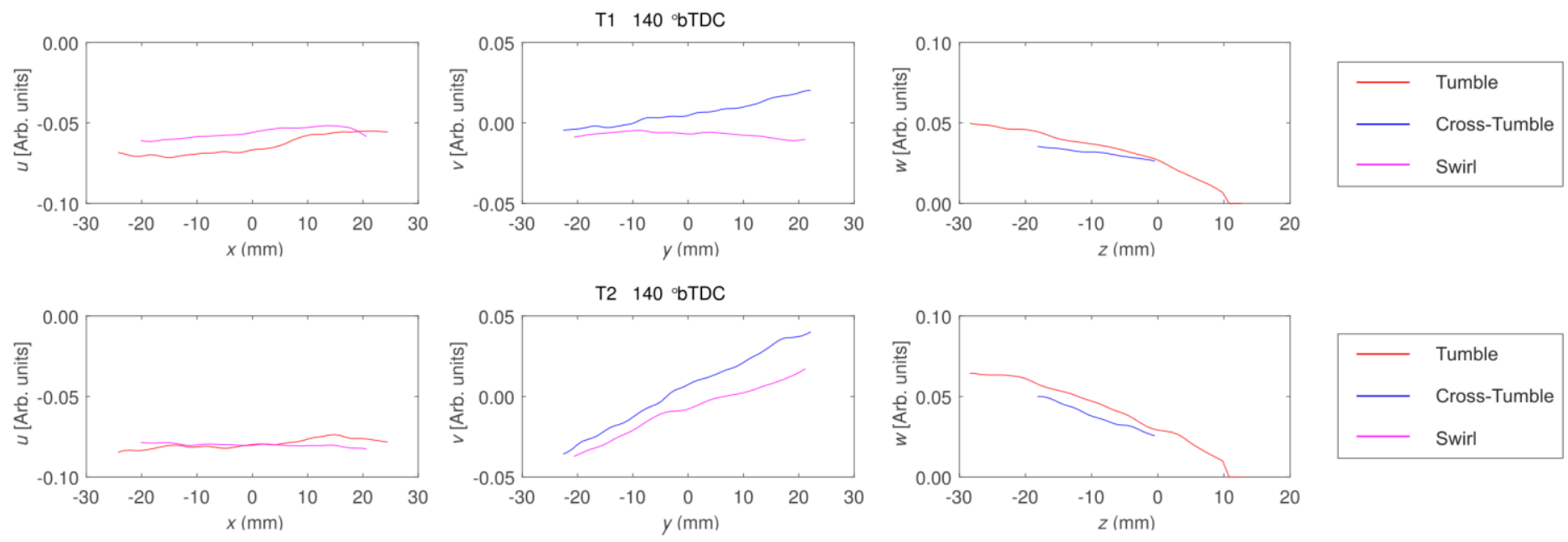

Figure 7 Comparison of common-component PIV velocities along the intersections of the three planes (Tumble, Cross-Tumble, Swirl) at $140^{\circ} \mathrm{bTDC}$.

\section{Initial Investigation Using the CMRI}

The WRI, WMI and CMRI metrics are used to identify key timings within the cycle for the $\mathrm{T} 1$ and $\mathrm{T} 2$ test conditions for which differences between the CFD and PIV flow fields are significant in one or more of the tumble, cross-tumble or swirl planes.

A wider set of four test points, of which two (T1 and T2) are discussed in this paper, were used for the normalisation of the WRI and WMI metric values to produce the CMRI. The differences between CFD and PIV for T1 and T2 are therefore quantified on a comprehensive scale that covers the variation found across all 4 test points and all 3 planes. This allows quantitative comparison of differences both between planes and between test points via the absolute values of the comparison metrics.
Figure 8 presents the spatially averaged CMRI values for the comparison of PIV and CFD flow fields throughout the intake and compression strokes for the $\mathrm{T} 1$ and $\mathrm{T} 2$ test conditions. This coarse overview condenses the spatial information within the comparison of PIV to CFD across each plane, for both directional and magnitude differences, into a robust scalar value for each crank angle. The CMRI values for each of the three planes are weighted by $1 / 3$ and displayed cumulatively at each crank angle as contributions to the 3 plane mean CMRI. In this way the height of the total shaded region represents the overall difference between PIV and CFD across all planes. The contribution of the tumble, cross-tumble and swirl planes to the combined difference in flow fields is given by the height of the blue, red and yellow regions respectively. 


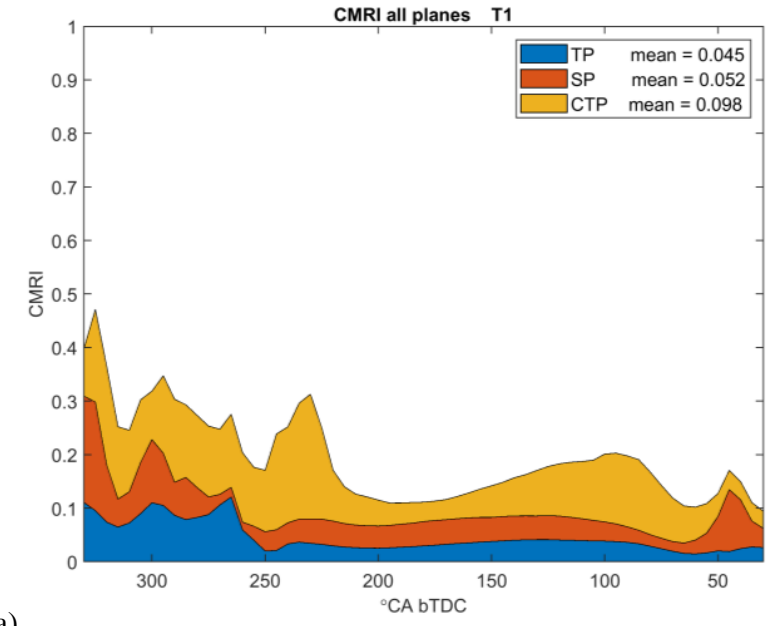

a)

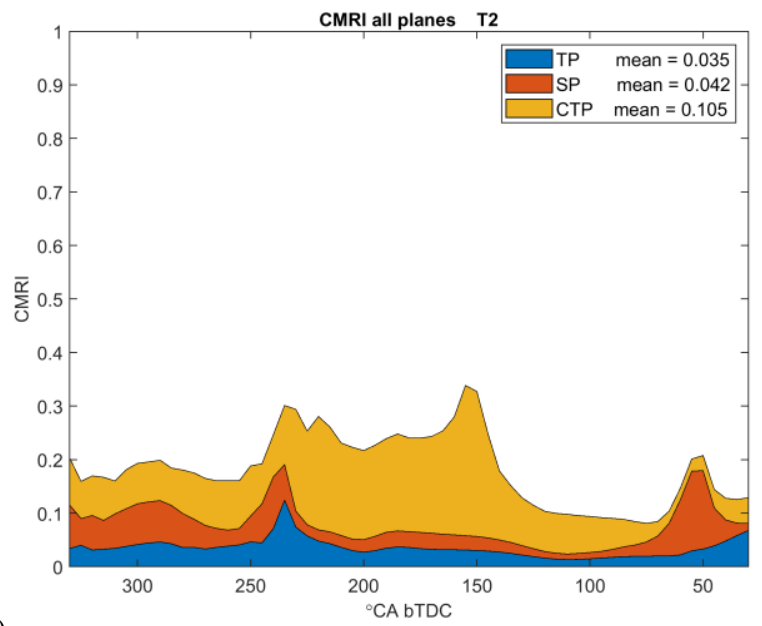

b)

Figure 8 Differences in both magnitude and direction between PIV and CFD flow fields as a function of crank angle for test conditions a) T1 and b) T2, quantified by spatially averaged CMRI values. Cumulative contributions to the mean CMRI value across all three planes are shown for the tumble plane (TP, blue), cross-tumble plane (CTP, yellow) and swirl plane (SP, red).

The cumulative CMRI values of Figure 8 identify five key timings during the intake and compression strokes which merit further investigation into the quality of the match between the CFD and PIV flow fields.

a) Quality of match across crank angles

i) Both test points are well matched overall, but T2 provides a slightly better match (lower CMRI) averaged over entire crank angle range $(0.183)$ compared to T1 (0.195).

ii) The cross-tumble plane provides the largest contribution to the differences across all crank angles by a factor of approximately 2 for both $\mathrm{T} 1$ $(\mathrm{CMRI}=0.098)$ and $\mathrm{T} 2(\mathrm{CMRI}=0.105)$.

iii) The tumble plane and swirl plane are similarly well matched, but with $\mathrm{T} 2$ better matched for both tumble plane and swirl plane (CMRI = 0.035 and 0.042$)$ than $\mathrm{T} 1(\mathrm{CMRI}=0.045$ and $0.052)$. b) Intake process

i) $\quad \mathrm{T} 2$ is better matched during intake $(\mathrm{CMRI} \approx 0.2)$.

1) Stable CMRI until rise during IVC (peak at $235^{\circ}$ bTDC).

2) Overall IVC peak in CMRI is due to differences in all three planes, where CMRI approximately doubles in each plane compared to preceding values (5$10^{\circ} \mathrm{CA}$ earlier) N.B. contributions peak across a $10^{\circ} \mathrm{CA}$ range (SP 240, TP 235, CTP $230^{\circ}$ bTDC) resulting in a more gradual overall rise in the mean CMRI values across all planes.

ii) $\mathrm{T} 1$ has greater differences during intake $(\mathrm{CMRI} \approx 0.3)$.

1) Variable CMRI throughout the intake until the match improves after IVC (local peak at $235^{\circ} \mathrm{bTDC}$ ).

2) Swirl plane is well-matched by IVC $(\mathrm{CMRI}=0.018)$ but large differences are identified for the tumble $(\mathrm{CMRI}=$ $0.121)$ and cross-tumble (CMRI = 0.137) planes.

c) Development of tumble flow

i) T1 has a peak in CMRI (0.313) around $230^{\circ}$ bTDC due to differences in the cross-tumble plane but is then well matched $(0.11<\mathrm{CMRI}<$ 0.14 ) across all 3 planes from $210^{\circ} \mathrm{bTDC}$ to $150^{\circ} \mathrm{bTDC}$

ii) T2 displays a consistently high total CMRI (approx. 0.25) lasting for approximately $90^{\circ} \mathrm{CA}$ from IVC at $230^{\circ}$ bTDC arising from differences in the cross-tumble plane. The swirl plane for $\mathrm{T} 2$ $(\mathrm{CMRI} \approx 0.025)$ is better matched than $\mathrm{T} 1$ $(\mathrm{CMRI} \approx 0.04)$, while the tumble plane is similarly well matched for both test conditions (both $\mathrm{CMRI} \approx 0.03$ ).

d) Mid-compression flow

i) In the cross-tumble plane, $\mathrm{T} 1$ displays a gradual increase in CMRI values from $160^{\circ} \mathrm{bTDC}$, peaking at $100^{\circ} \mathrm{bTDC}$. In contrast, once the high CMRI plateau of T2 ends at around $140^{\circ} \mathrm{bTDC}$, there is a gradual decrease in CMRI within the cross-tumble plane for $\mathrm{T} 2$.

ii) T2 has an excellent match in the tumble $(\mathrm{CMRI}=0.016)$ and swirl $(\mathrm{CMRI}=0.015)$ planes. Major contribution arises from differences in the cross-tumble plane $(\mathrm{CMRI}=0.063)$.

iii) $\mathrm{T} 1 \mathrm{has}$ a good match in the tumble $(\mathrm{CMRI}=0.038)$ and the swirl $(\mathrm{CMRI}=0.033)$ planes. Major contribution to poorer match than $\mathrm{T} 2$ arises from differences in cross-tumble plane $(\mathrm{CMRI}=0.132)$.

e) Late-compression flow

i) Both $\mathrm{T} 1$ and $\mathrm{T} 2$ have a peak in CMRI around $50^{\circ}$ bTDC solely due to differences within the swirl plane.

The spatially averaged CMRI results already give a quantitative overview of where the CFD flow fields matches well with PIV for each test condition. However far more information is available, firstly by further investigation using the WRI and WMI metrics to 
distinguish between differences in alignment from differences in magnitude, and secondly using the key timings of interest identified for each plane to inform the inspection of the spatially resolved metric fields and the flow fields themselves.

The key features of the CMRI values in Figure 8 can be assigned as arising from two main characteristics of the in-cylinder flow:

b) The development of the intake jet formed while the inlet valves are open.

c), d) and e) The motion of the tumble vortex center throughout the cylinder for this tumble-dominated flow geometry.

Both characteristics are highly three-dimensional flow structures and their dynamics are best studied by consideration of the flow field interactions across all three planes.

\section{Intake process}

\section{Tumble plane $-\mathrm{T} 1$ - 3 peaks in CMRI}

The CMRI values of T1 in Figure 8 show three distinct peaks at $330^{\circ} \mathrm{bTDC}, 300^{\circ} \mathrm{bTDC}$ and $265^{\circ} \mathrm{bTDC}$ during intake for the tumble plane, and these correspond to significant differences in the simulated and experimental flow fields. Investigation of the flow fields at these key timings [Figure 9] reveals these CMRI peaks arise from different features of the intake flow fields.

1) $330^{\circ} \mathrm{bTDC}$ Jet direction and magnitude

2) $300^{\circ}$ bTDC Tumble cross-flow PIV only

3) $265^{\circ} \mathrm{bTDC}$ At IVC PIV jet disappears earlier, CFD jet sharpens

At $330^{\circ}$ bTDC the local flow directions are well matched with low (blue) WRI values but there is a large difference in the velocity magnitudes with high (yellow) WMI values which leads to the first CMRI peak. The high velocity region of the PIV intake jet is located further towards the exhaust while the center of the intake jet predicted by CFD is closer to the inlet and has a higher velocity than the jet measured by PIV.
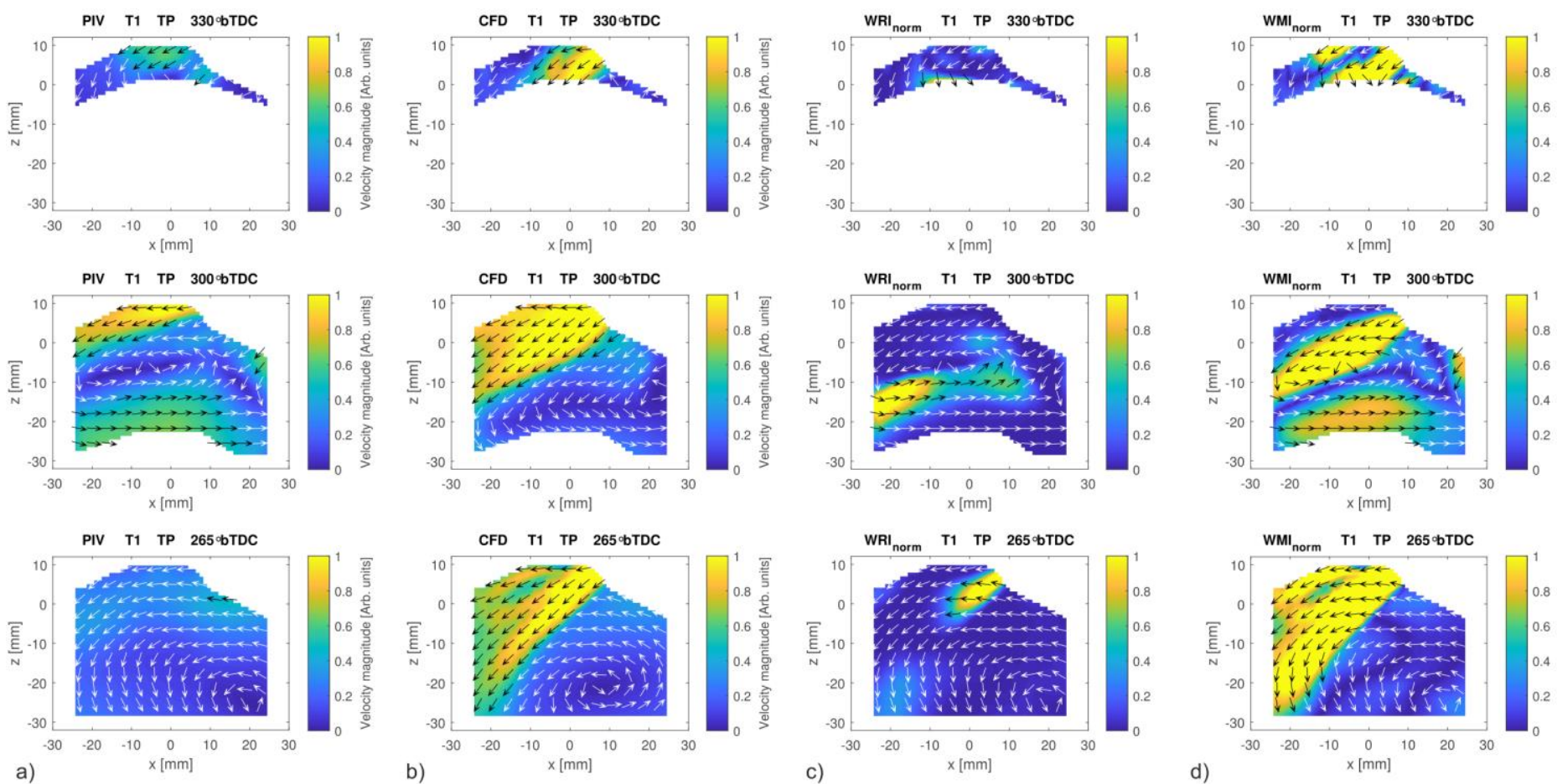

Figure 9 a) Experimental PIV and b) simulated CFD flow fields during the intake process for T1 with local differences in c) direction and d) magnitude quantified using the WRI and WMI metrics respectively. Each row displays data from a different crank angle identified as a key timing of interest by a peak in the CMRI values of Figure 8. For clarity only every fourth vector is plotted in each direction, with a color (black/white) chosen to contrast with the false color background.

By $300^{\circ}$ bTDC the intake jet for both PIV and CFD is well established. The CFD jet takes up a much larger region of the upperexhaust side of the field of view with a clear distinction between the high velocity jet and the slow bulk flow in the central and inlet side regions of the cylinder. The PIV jet is located much closer to the cylinder head and the overlap between the slow recirculating flow below the PIV jet and the fast lower edge of the CFD jet produces very high WMI values. The PIV flow field also contains a $15-20$ $\mathrm{mm}$ high medium velocity horizontal flow across the piston. This cross-flow generates high WRI values on the exhaust side where it opposes the CFD intake jet direction and high WMI values above the piston surface where the simulated flow is much lower velocity.

As the intake valves close, the behaviour of the intake jet differs between the PIV and CFD flow fields. For PIV, the closing valves result in the disappearance of the intake jet at $265^{\circ}$ bTDC when the valve lift is very small and the formation of the bulk tumble flow which persists for the remainder of the cycle. At the same crank angle, the small valve lift results in a narrowing of the intake jet and an increase in peak velocity for the CFD flow field. The CFD intake 
jet disappears once the valves are fully closed at $255^{\circ} \mathrm{bTDC}$, regaining a good match with the PIV flow fields.

\section{Tumble plane - T2 - IVC peak only}

In contrast, the higher load $\mathrm{T} 2$ has a very good overall match while the intake valve are open [Figure 10]. For both $330^{\circ} \mathrm{bTDC}$ and $300^{\circ}$ bTDC there is an excellent directional match between PIV and CFD, with only a minor overestimation of the velocity magnitudes for CFD resulting in slightly raised WMI values. The intake jet is
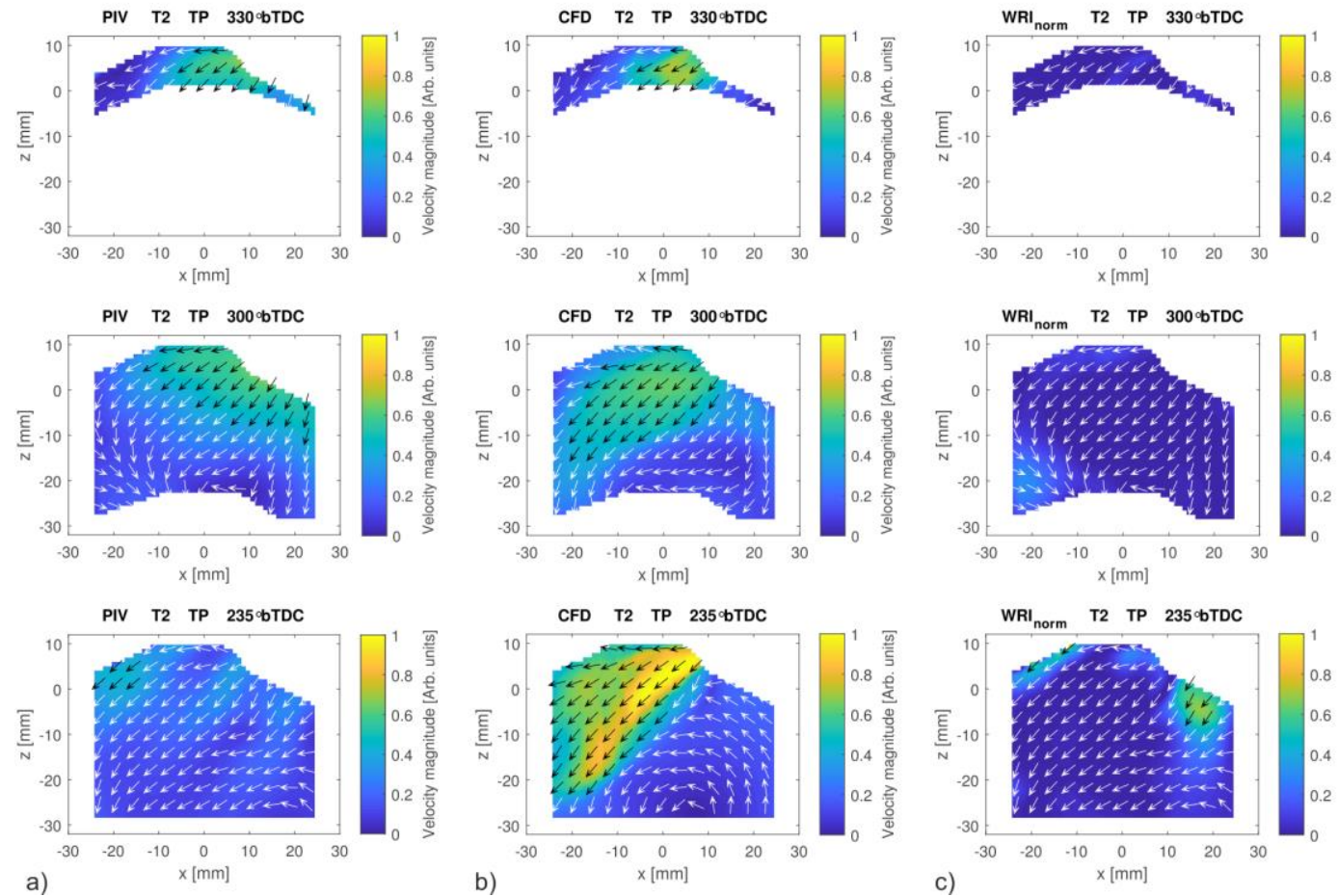

much broader and lower velocity than T1, covering a large portion of the field of view and does not result in the cross-piston flow present in the T1 PIV flow field.

Once the intake valves have almost fully closed at $235^{\circ}$ bTDC $(15 \%$ of peak lift) however, a similar behaviour to that of T1 is observed. The PIV intake jet disappears and the CFD intake jet again narrows and increases in peak velocity magnitude, resulting in high WMI values followed by the formation of bulk tumble flow at $225^{\circ}$ bTDC.

Figure 10 a) Experimental PIV and b) simulated CFD flow fields during the intake process for T2 with local differences in c) direction and d) magnitude quantified using the WRI and WMI metrics respectively. Each row displays data from a different crank angle identified as a key timing of interest by a peak in the CMRI values of Figure 8 . For clarity only every fourth vector is plotted in each direction, with a color (black/white) chosen to contrast with the false color background.

\section{Tumble vortex motion}

\section{Development of tumble flow $-\mathrm{T} 1-10^{\circ} \mathrm{CA}$ delay in vortex crossing}

The CMRI peak for $\mathrm{T} 1$ around $230^{\circ} \mathrm{bTDC}$ arises from the tumble vortex center passing through the cross-tumble measurement plane $10^{\circ} \mathrm{CA}$ earlier for the PIV measured flow field compared to the CFD simulation [Figure 11]. The low velocities near the tumble vortex center first make an appearance in the PIV flow fields at $250^{\circ} \mathrm{bTDC}$, disturbing the otherwise downwards flow in the central tumble plane as the tumble vortex center is initially formed on the inlet side of the cylinder and the tumble motion is anti-clockwise when viewed in the tumble plane of Figure 9.
As the tumble vortex center traverses from inlet to exhaust, the flow in the central cross-tumble reverses for PIV at $240^{\circ} \mathrm{bTDC}$ resulting in an upwards flow for $230^{\circ} \mathrm{bTDC}$ and $220^{\circ} \mathrm{bTDC}$.

This vortex crossing occurs $10^{\circ} \mathrm{CA}$ later for the CFD flow field at $230^{\circ} \mathrm{bTDC}$, causing first high values of the WRI due to the opposed flows between CFD and PIV at $240^{\circ}$ bTDC, followed by high values of the WMI due to the low velocities within the tumble vortex center for CFD at $230^{\circ} \mathrm{bTDC}$. Once the tumble vortex is found on the exhaust side of the cylinder for both PIV and CFD there is a good match in both direction and magnitude from $220^{\circ} \mathrm{bTDC}$ to $150^{\circ} \mathrm{bTDC}$ as shown by the low CMRI values in Figure 8. 

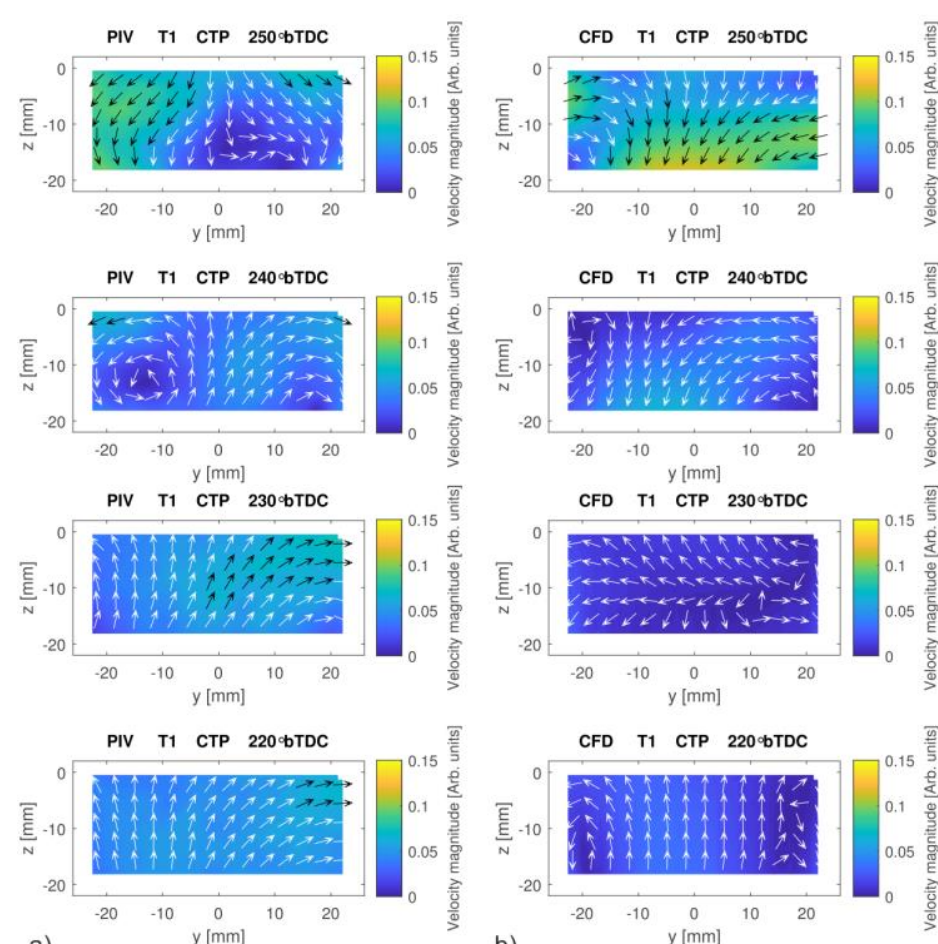

a)

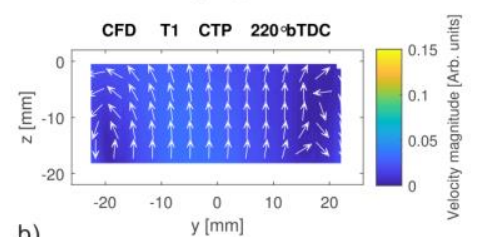

b)
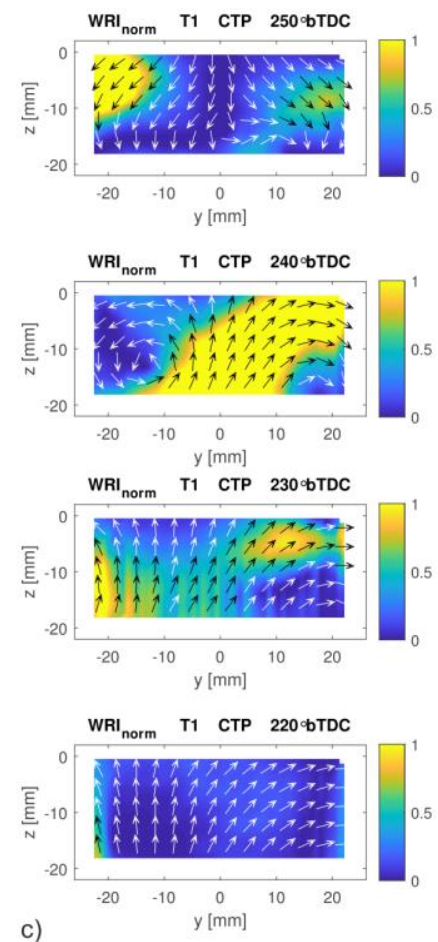

c)
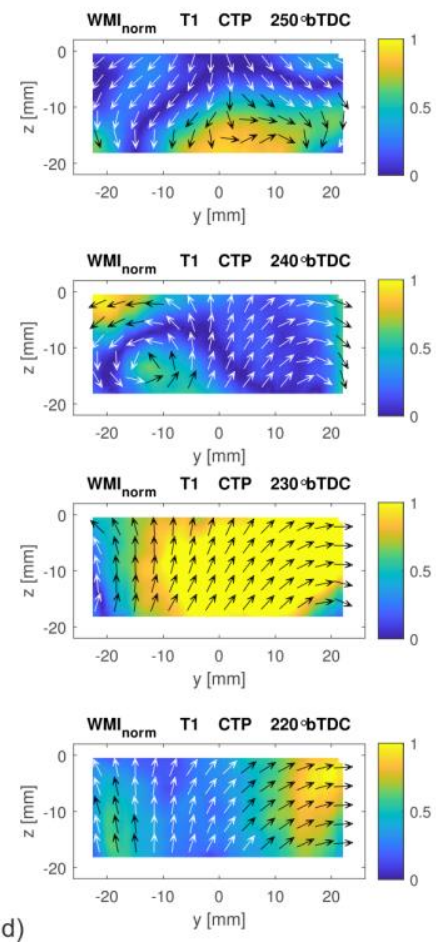

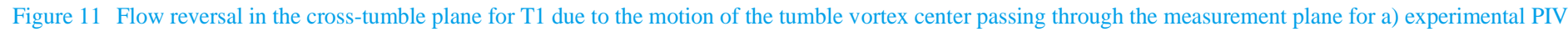

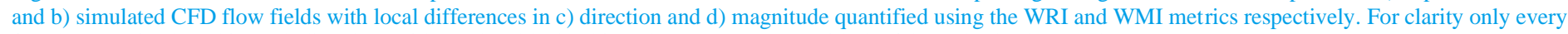
fourth vector is plotted in each direction, with a color (black/white) chosen to contrast with the false color background.

\section{Development of tumble flow - T2 - CTP flow reversals due to vortex crossing and TP slow flow region}

The extended period of high spatially averaged CMRI values in the cross-tumble plane from $230^{\circ} \mathrm{bTDC}$ to $140^{\circ} \mathrm{bTDC}$ identified in Figure 8 may be assigned to a difference primarily in flow direction as high WRI values provide the dominant contribution to the increase in CMRI over these crank angles [Figure 12b]. These directional differences arise from a sequence of flow reversals for both PIV and CFD in the cross-tumble plane as a result of the motion of the tumble vortex center across the tumble plane during the cycle [Figure 13].

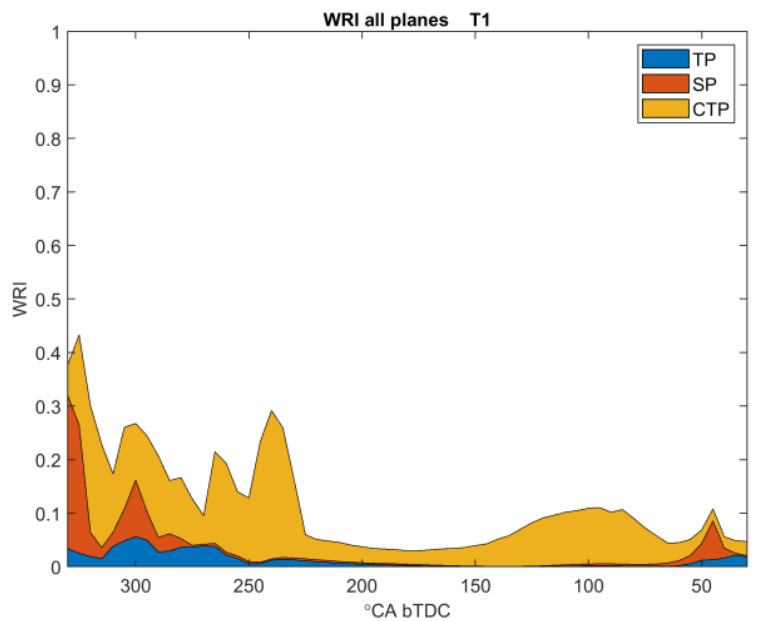

At $225^{\circ} \mathrm{bTDC}$ the intake valves are fully closed and the bulk tumble flow is establishing. The tumble vortex center for the CFD flow field is at approximately $\mathrm{z}=-30 \mathrm{~mm}$ and the tumble motion follows the sloped geometry of the cylinder head giving an upwards flow in the cross-tumble plane. For the tumble plane PIV flow field the tumble vortex center is located below the field of view and a central region of slow flow near the cylinder head diverts the tumble flow downwards into the center of the cylinder. This causes the downwards flow in the PIV cross-tumble plane and corresponding high WRI values for the opposed PIV and CFD flow directions. The presence of this slow flow region is also consistent with a similar observation in earlier work without cross-tumble or swirl data with a different piston crown geometry [23].

a)

b)

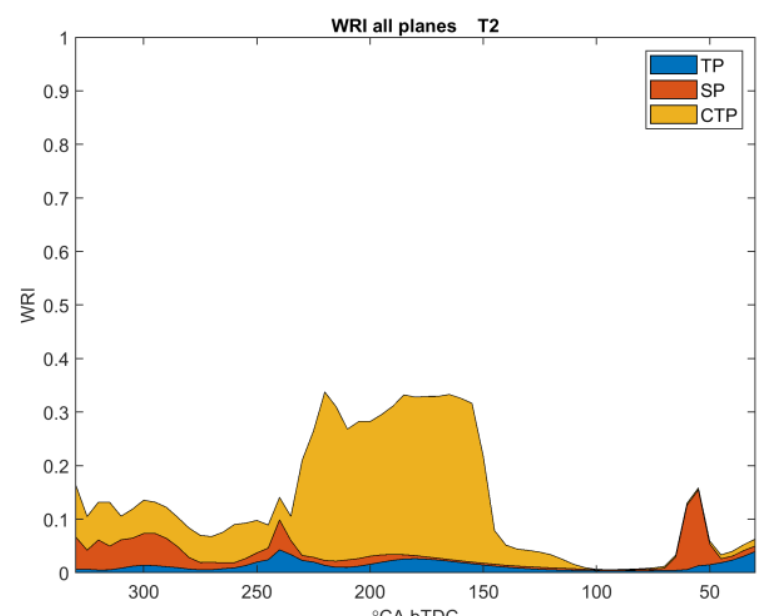

Figure 12 Differences in direction between PIV and CFD flow fields as a function of crank angle for test conditions a) T1 and b) T2, quantified by spatially averaged WRI values. Cumulative contributions to the mean WRI value across all three planes are shown for the tumble plane (TP, blue), cross-tumble plane (CTP, yellow) and swirl plane (SP, red).

Page 11 of 17 

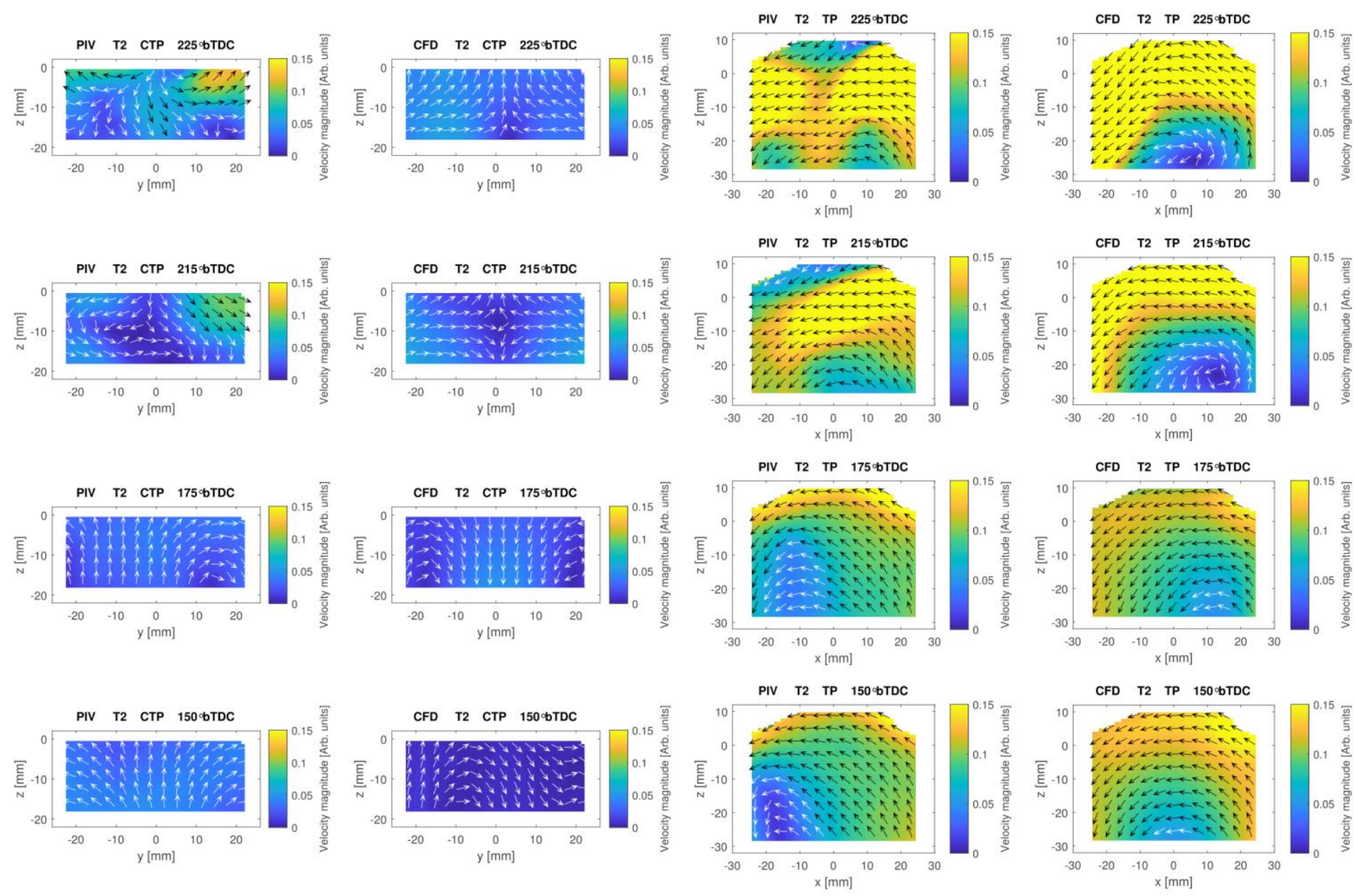

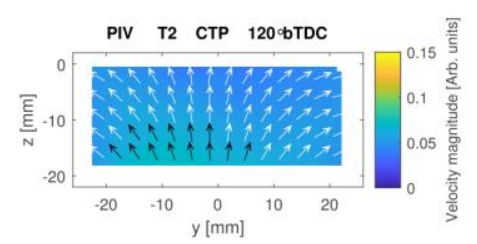

a)

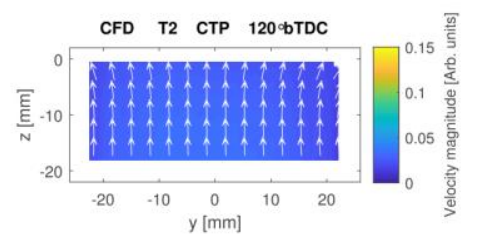

b)

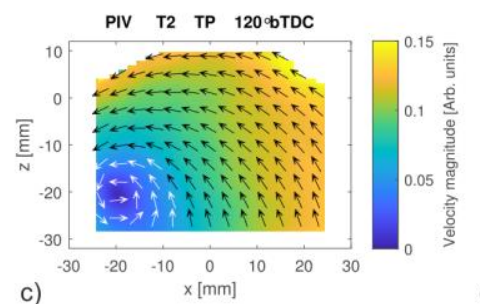

c)

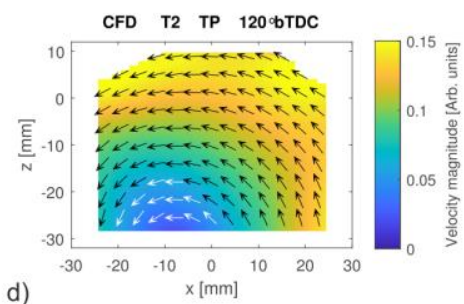

d)

Figure 13 Flow reversals in the cross-tumble plane for T2 arising from the tumble vortex center crossing from inlet to exhaust during late intake and early

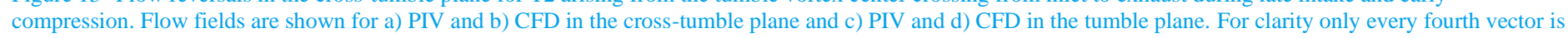
plotted in each direction, with a color (black/white) chosen to contrast with the false color background.

At $215^{\circ} \mathrm{bTDC}$ the tumble vortex center moves towards the inlet for CFD and towards the exhaust for PIV resulting in a synchronised switch in cross-tumble plane flow direction which maintains the opposed flows. These high WRI values persist while the tumble vortex centers of the PIV and CFD flow fields lie on opposite sides of the cylinder. It is not until $150^{\circ} \mathrm{bTDC}$ when the CFD tumble vortex center traverses to the exhaust side of the cylinder and reverses the CFD cross-tumble flow, that the WRI values improve. By $140^{\circ}$ bTDC both PIV and CFD have an upwards flow direction in the crosstumble plane and this good match (low WRI values) is maintained for the rest of the compression stroke [Figure 12b].

\section{Asymmetric CFD flow during compression - T1 cross- tumble plane}

Aside from the timings of flow reversals, the cross-tumble flow fields between IVC and $160^{\circ}$ bTDC display nearly uniform vertical flow directions for both T1 [Figure 11] and T2 [Figure 13].
For T2, once the final flow reversal occurs at $150^{\circ} \mathrm{bTDC}$ for CFD, the CMRI values show a good match across all 3 planes until $70^{\circ}$ bTDC [Figure 8], with vertical flow direction in the cross-tumble plane [Figure 13]. In contrast, after $160^{\circ} \mathrm{bTDC}$ the cross-tumble plane CMRI values for $\mathrm{T} 1$ increase to peak at $100^{\circ} \mathrm{bTDC}$ [Figure 8] as a result of increased WRI values [Figure 12a]. Over the same region, there is an excellent match between CFD and PIV for T2 with nearzero WRI values [Figure 12b].

The increased WRI values for T1 are due to the appearance of asymmetry in the CFD flow field which is not observed in the PIV flow field [Figure 14]. 

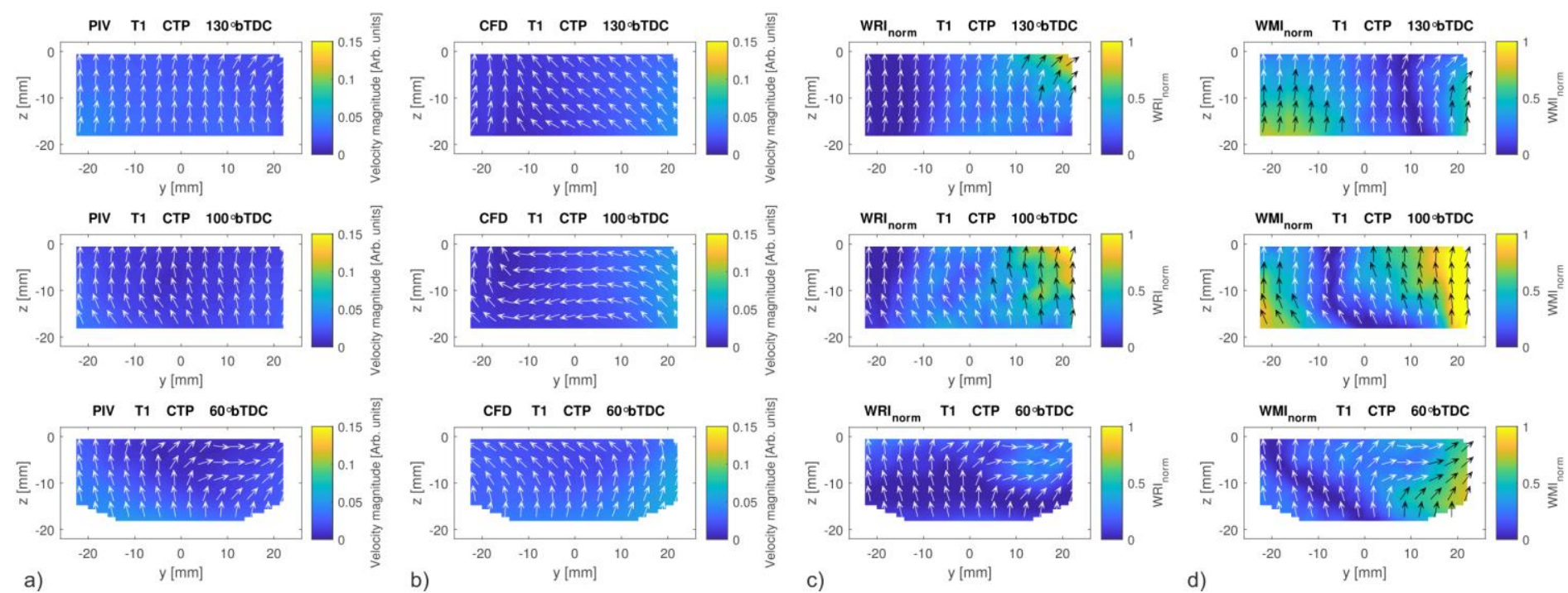

Figure 14 Asymmetric mid-compression cross-tumble flow field feature for T1 for a) experimental PIV and b) simulated CFD flow fields with local differences in c) direction and d) magnitude quantified using the WRI and WMI metrics respectively. For clarity only every fourth vector is plotted in each direction, with a color (black/white) chosen to contrast with the false color background.

At $160^{\circ} \mathrm{bTDC}$ the cross-tumble flow field for $\mathrm{T} 1$ begins to differ from the vertical PIV flow field. By $130^{\circ} \mathrm{bTDC}$, in comparison to the previously well-matched PIV flow field, the CFD velocities on the right of the cross-tumble plane increase in magnitude and rotate towards the left of the cross-tumble plane where the CFD velocity magnitude is reduced [Figure 14]. At $100^{\circ}$ bTDC the CFD flow field has become horizontal across the central $20 \mathrm{~mm}$ of the cross-tumble plane producing high WRI values when compared to the PIV flow field which remains vertical, similarly to the PIV and CFD flow fields of T2 [Figure 13].

This difference in flow characteristic persists until $60^{\circ} \mathrm{bTDC}$ when the rising piston causes the region of horizontal flow in CFD to leave the cross-tumble field of view, to be replaced by vertical flow near the piston which is well matched to the PIV flow field [Figure 14].

\section{Swirl plane vortex crossing - T1 and T2}

Towards the end of the compression stroke, the flow fields in the tumble and cross-tumble planes are well matched for both T1 and T2 with spatially averaged CMRI values ranging from 0.020 to 0.043 at $50^{\circ}$ bTDC [Figure 8].

The swirl plane CMRI values display a peak at $45^{\circ} \mathrm{bTDC}$ for $\mathrm{T} 1$ and at $55^{\circ} \mathrm{bTDC}$ for $\mathrm{T} 2$. Inspection of the swirl plane flow fields identifies a delayed flow reversal for CFD compared to PIV as the tumble vortex center is carried through the swirl measurement plane by the rising piston compressing the in-cylinder flow [Figure 15].

Initially the tumble vortex center for both PIV and CFD lies below the swirl measurement plane near the cylinder axis [Figure 4]. The swirl plane flow at $\mathrm{z}=-3.5 \mathrm{~mm}$ is therefore from inlet to exhaust for the majority of the intake and compression strokes. For T1, at $50^{\circ} \mathrm{bTDC}$ a pair of counter-rotating vortices appear in the PIV swirl plane flow field for $\mathrm{T} 1$ at approximately $\mathrm{y}= \pm 10 \mathrm{~mm}$ [Figure 15]. Similar vortices appear in the CFD flow field $5^{\circ} \mathrm{CA}$ later at $45^{\circ} \mathrm{bTDC}$, at which time the PIV flow field has reversed to be directed from exhaust to inlet. The PIV tumble vortex center has passed through the swirl measurement plane, leaving the PIV to measure the horizontal components of velocity in the lower half of the tumbling flow. Within $5^{\circ} \mathrm{CA}$ the CFD flow field also reverses, recovering the good match in swirl plane direction and magnitude found throughout the majority of the cycle [Figure 8].

For T2 the flow field also reverses first for PIV at $60^{\circ} \mathrm{bTDC}$ but with a $10^{\circ}$ bTDC delay before the CFD flow field reverses at $50^{\circ} \mathrm{bTDC}$ [Figure 16]. Instead of a pair of counter-rotating vortices, the flow reverses in the central region of the swirl plane, surrounded by an annulus of low in-plane velocities. This may be linked to the offset in the $\mathrm{T} 2$ tumble vortex center location approximately $20 \mathrm{~mm}$ towards the exhaust compared to the central location for T1 [Figure 5]. 

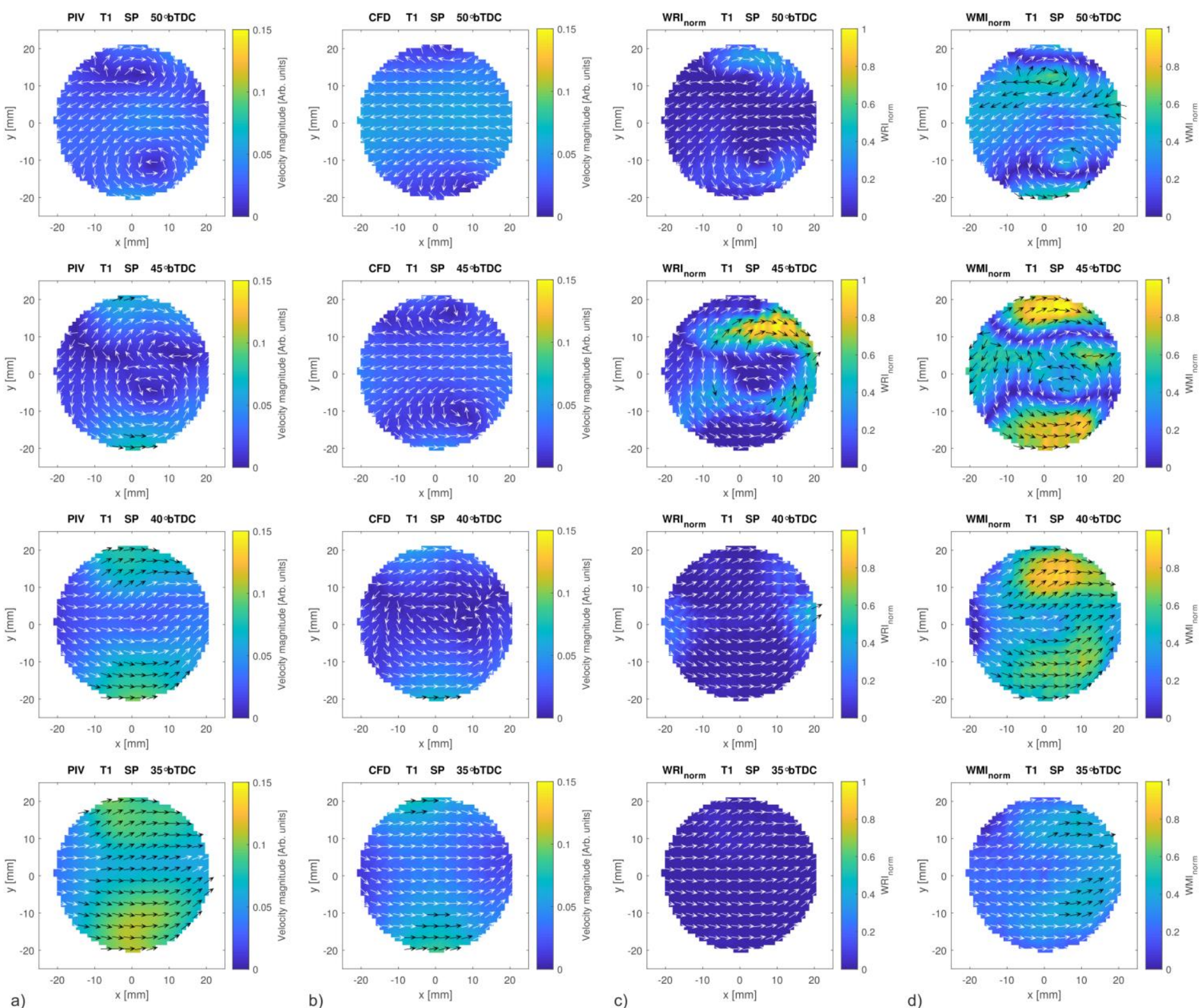

Figure 15 Delay between PIV and CFD flow reversal in T1 depending on when the tumble vortex passes through the swirl measurement plane for a) experimental PIV and b) simulated CFD flow fields with local differences in c) direction and d) magnitude quantified using the WRI and WMI metrics respectively. For clarity only every third vector is plotted in each direction, with a color (black/white) chosen to contrast with the false color background. 

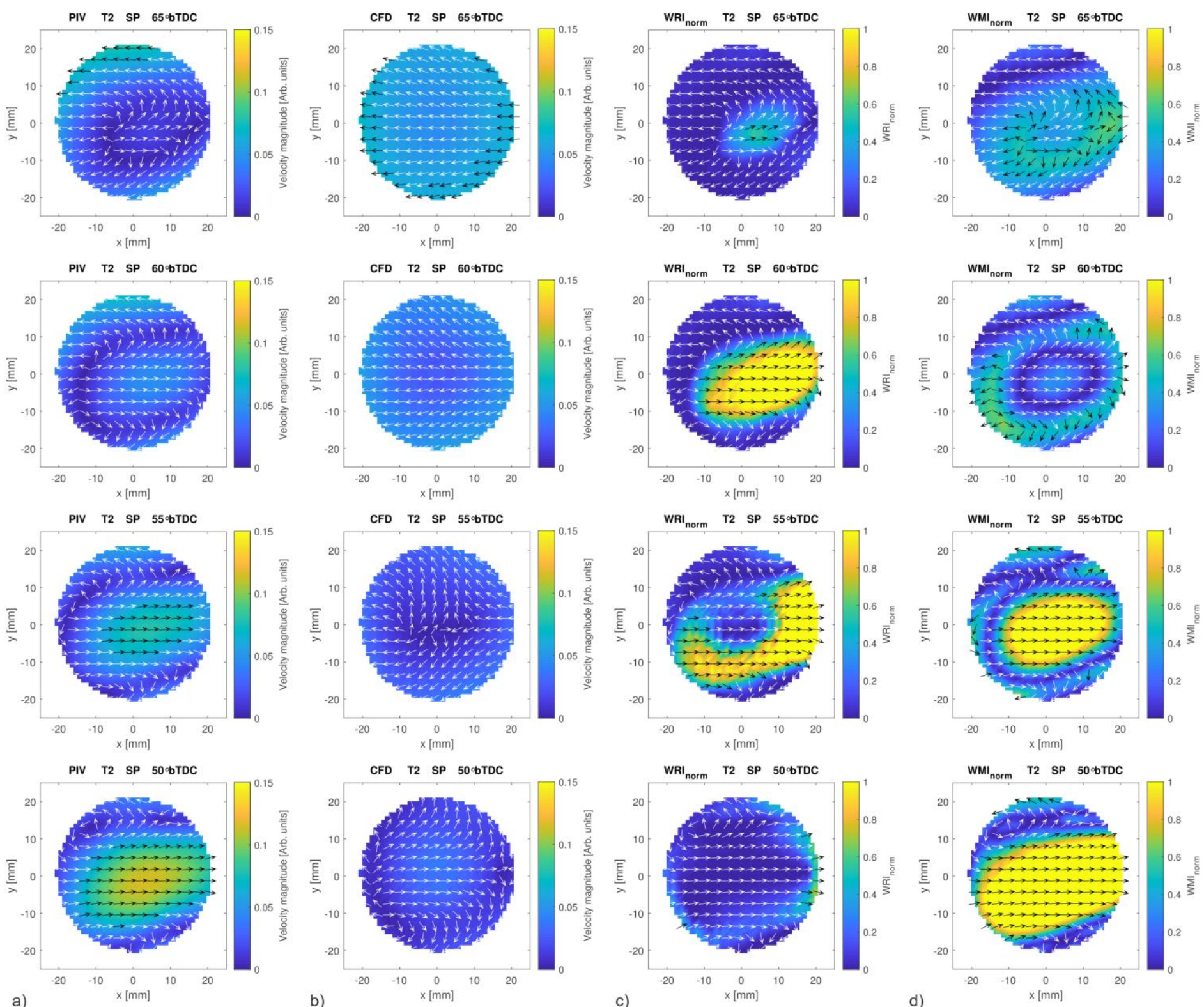

Figure 16 Delay between PIV and CFD flow reversal in T2 as the tumble vortex passes through the swirl measurement plane at different times for a) experimental PIV and b) simulated CFD flow fields with local differences in c) direction and d) magnitude quantified using the WRI and WMI metrics respectively. For clarity only every third vector is plotted in each direction, with a color (black/white) chosen to contrast with the false color background.

\section{Conclusions}

High speed PIV measurements of the in-plane velocities across the tumble, cross-tumble and swirl planes of an optically accessible GDI engine have been quantitatively compared to RANS simulations of the flow fields for motored operation. Spatially-resolved differences in alignment and magnitude between the experimental and simulated in-cylinder flow fields are quantified both independently using the Weighted Relevance Index (WRI) and Weighted Magnitude Index (WMI), as well as in combination using the Combined Magnitude and Relevance Index (CMRI).

Spatially averaged CMRI values for each plane provide a rapid and quantitative method for evaluating the quality of match between PIV and CFD flow fields both between planes and between test conditions. Overall the higher load test condition (T2) provided the best match between simulation and experiment.
Application of the CMRI, WRI and WMI metrics also enabled the identification of key timings within the intake and compression strokes, specific to each test point, for further investigation into differences between the PIV and CFD flow fields. In general, a very good match was found between the PIV and CFD flow fields across all three planes and for both test conditions. Differences that were identified include:

- The RANS simulation accurately captures the intake flow characteristics for $\mathrm{T} 2$ but overpredicts the velocities within the intake jet for the lower load test point (T1). For both test points the increased jet velocities predicted by CFD during inlet valve closing were not observed for PIV.

- A series of flow direction reversals in the cross-tumble and swirl planes arise due to the development of the bulk tumble motion and the subsequent motion of the tumble vortex center during the cycle. Differences in the location of the tumble vortex center 
between the PIV and CFD flow fields resulted in delays of 5 to $10^{\circ} \mathrm{CA}$ in the in-plane flow reversals for CFD compared to PIV.

- A low velocity asymmetric flow in the cross-tumble plane was predicted by CFD during compression for T1 but not observed in the PIV measurements which showed a uniform vertical flow for the corresponding crank angles.

In this work the ensemble mean PIV fields, each derived from, 300 cycles, are compared to the RANS simulated flow fields. As such, the effect of cycle to cycle fluctuations and turbulence present in each individual PIV cycle is averaged out, resulting in mean velocities which are less extreme than those found in an individual cycle. Indeed, where differences in magnitude are present, the RANS velocity magnitudes for $\mathrm{T} 1$ and $\mathrm{T} 2$ are typically higher than the corresponding mean PIV magnitudes. A representative single cycle may provide a more appropriate comparison to RANS flow fields and is the subject of a future study.

\section{Acknowledgements}

The authors would like to thank Jaguar Land Rover Ltd for financial and technical support and thank EPSRC and Innovate UK [project 63697-464157] for partial financial support. Li Shen is grateful for the grants from the Henry Lester Trust.

\section{References}

[1] G. Kalghatgi, "Is it really the end of internal combustion engines and petroleum in transport?," Appl. Energy, vol. 225, pp. 965-974, 2018.

[2] N. O. Kapustin and D. A. Grushevenko, "Long-term electric vehicles outlook and their potential impact on electric grid," Energy Policy, 2019.

[3] W. Hentschel, B. Block, T. Hovestadt, H. Meyer, G. Ohmstede, V. Richter, B. Stiebels, and A. Winkler, "Optical Diagnostics and CFD-Simulations to Support the Combustion Process Development of the Volkswagen FSI ${ }^{\circledR}$ Direct-Injection Gasoline Engine," SAE Technical Paper 2001-01-3648, 2001.

[4] T. W. Kuo, X. Yang, V. Gopalakrishnan, and Z. Chen, "Large Eddy Simulation (LES) for IC Engine Flows," Oil Gas Sci. Technol., vol. 69, no. 1, pp. 61-81, 2014.

[5] T. Wang, W. Li, M. Jia, D. Liu, W. Qin, and X. Zhang, "Large-eddy simulation of in-cylinder flow in a DISI engine with charge motion control valve: Proper orthogonal decomposition analysis and cyclic variation," Appl. Therm. Eng., vol. 75, pp. 561-574, 2015.

[6] B. Böhm, C. Heeger, R. L. Gordon, and A. Dreizler, "New perspectives on turbulent combustion: Multi-parameter highspeed planar laser diagnostics," Flow, Turbul. Combust., vol. 86, no. 3-4, pp. 313-341, 2011.

[7] A. K. Prasad, "Stereoscopic particle image velocimetry," Exp. Fluids, vol. 29, pp. 103-116, 2000.

[8] R. J. Adrian and J. Westerweel, Particle image velocimetry. Cambridge: Cambridge University Press, 2011.
B. Peterson, E. Baum, C. P. Ding, D. Michaelis, A. Dreizler, and B. Böhm, "Assessment and application of tomographic PIV for the spray-induced flow in an IC engine," Proc. Combust. Inst., vol. 36, no. 3, pp. 3467-3475, 2017.

[10] M. Raffel, C. E. Willert, F. Scarano, C. Kähler, S. T. Wereley, and J. Kompenhans, Particle Image Velocimetry: A Practical Guide, 3rd ed. Springer International Publishing, 2018.

[11] T. Justham, S. Jarvis, C. P. Garner, G. K. Hargrave, A. Clarke, and D. Richardson, "Single cylinder motored SI IC engine intake runner flow measurement using time resolved digital particle image velocimetry," SAE Tech. Pap., 200601-1043. 2006,

[12] W. Zeng, M. Sjöberg, and D. Reuss, "Using PIV Measurements to Determine the Role of the In-Cylinder Flow Field for Stratified DISI Engine Combustion," SAE Int. J. Engines, vol. 7, no. 2, pp. 615-632, 2014.

[13] W. Zeng, M. Sjöberg, D. L. Reuss, and Z. Hu, "The role of spray-enhanced swirl flow for combustion stabilization in a stratified-charge DISI engine," Combust. Flame, vol. 168, no. $x$, pp. 166-185, 2016.

[14] M. Zhang, M. Xu, and D. L. S. Hung, "Simultaneous twophase flow measurement of spray mixing process by means of high-speed two-color PIV," Meas. Sci. Technol., vol. 25, no. 9 , p. 095204, 2014.

[15] R. Stiehl, J. Bode, J. Schorr, C. Krüger, A. Dreizler, and B. Böhm, "Influence of intake geometry variations on incylinder flow and flow-spray interactions in a stratified direct-injection spark-ignition engine captured by timeresolved particle image velocimetry," Int. J. Engine Res., vol. 17, no. 9, pp. 983-997, Mar. 2016.

[16] F. W. Bowditch, "A New Tool for Combustion Research A Quartz Piston Engine.” SAE International Paper 610002, 1961.

[17] K. Liu and D. C. Haworth, "Development and Assessment of POD for Analysis of Turbulent Flow in Piston Engines," SAE Technical Paper 2011-01-0830, 2011.

[18] Y. Wang, D. L. S. Hung, H. Zhuang, and M. Xu, "Cycle-toCycle Analysis of Swirl Flow Fields inside a Spark- Ignition Direct-Injection Engine Cylinder Using High-Speed TimeResolved Particle Image Velocimetry," in SAE Technical Paper 2016-01-0637, 2016.

[19] H. Chen, H. Zhuang, D. L. Reuss, and V. Sick, "Influence of Early and Late Fuel Injection on Air Flow Structure and Kinetic Energy in an Optical SIDI Engine," SAE Technical Paper 2018-01-0205, 2018.

[20] B. Hu, S. Banerjee, K. Liu, and Q. Xue, "Large Eddy Simulation of a Turbulent Non-Reacting Spray Jet," in ASME 2015 Internal Combustion Engine Division Fall Technical Conference, 2015.

[21] F. Zhao, M. Liu, P. Ge, D. L. S. Hung, X. Li, M. Xu, X. Yang, and C. Idicheria, "Multi-plane time-resolved Particle Image Velocimetry (PIV) flow field measurements in an 
optical Spark-Ignition Direct-Injection (SIDI) engine for Large-Eddy Simulation (LES) model validations," Oil Gas Sci. Technol. - Rev. d'IFP Energies Nouv., vol. 74, p. 52, 2019.

[22] C. Willman, B. Scott, R. Stone, and D. Richardson, "Quantitative metrics for comparison of in-cylinder velocity fields using particle image velocimetry," Exp. Fluids, vol. 61 , no. 2, pp. 1-16, 2020.

[23] B. Scott, C. Willman, R. Stone, G. Virelli, R. Magnanon, and D. Richardson, "Novel metrics for validation of PIV and CFD in IC engines," SAE Technical Paper 2019-01-0716, 2019.

[24] P. Abraham, D. Reuss, and V. Sick, "High-Speed Particle Image Velocimetry Study of In-Cylinder Flows with Improved Dynamic Range," SAE Technical Paper 2013-010542, 2013.

[25] D. L. Reuss, M. Megerle, and V. Sick, "Particle-image velocimetry measurement errors when imaging through a transparent engine cylinder," Meas. Sci. Technol., vol. 13, pp. 1029-1035, 2002.

[26] A. K. Prasad, R. J. Adrian, C. C. Landreth, and P. W. Offutt, "Effect of Resolution on the Speed and Accuracy of Particle Image Velocimetry Interrogation," Exp. Fluids, vol. 13, pp. 105-116, 1992.

[27] J. Westerweel and F. Scarano, "Universal outlier detection for PIV data," Exp. Fluids, vol. 39, no. 6, pp. 1096-1100, 2005.

\section{Contact Information}

Christopher Willman

Engineering Science, Parks Road, OX1 3PJ

christopher.willman@eng.ox.ac.uk

\section{Definitions/Abbreviations}

\begin{tabular}{ll} 
aTDC & After TDC (firing) \\
bTDC & Before TDC (firing) \\
${ }^{\circ}$ Ca & Crank Angle Degrees \\
CFD & Computational Fluid Dynamics \\
CMRI & $\begin{array}{l}\text { Combined Magnitude and } \\
\text { GDI }\end{array}$ \\
ICE & Gasoline Direct Injection \\
IVC & Internal Combustion Engine \\
PIV & Inlet Valve Closing \\
RANS & Particle Image Velocimetry \\
RI & Reynolds averaged Navier-Stokes \\
TDC & Relevance Index \\
WMI & Top Dead Center \\
WRI & Weighted Magnitude Index \\
\hline
\end{tabular}

